\title{
Point prevalence survey of antimicrobial utilization in oncology patients
}

\author{
Arwa Hammuda ${ }^{1}$, Sara Hayder ${ }^{1}$, Shereen Elazzazy ${ }^{2}$, Emily Black ${ }^{1}$ \\ ${ }^{1}$ Qatar University, College of Pharmacy, Doha, Qatar \\ ${ }^{2}$ Hamad Medical Corporation, National Center for Cancer Care and Research, Doha, Qatar
}

\begin{abstract}
Introduction: Point prevalence surveys have been used internationally to characterize the use of antimicrobial agents. The primary objective of this study was to determine prevalence of antimicrobial use in the oncology population in Qatar.

Methodology: Data was collected from the National Center for Cancer Care and Research hospital over 3 separate days during a two week period. Adults admitted on the day of audit were included.

Results: Prevalence of antimicrobial use during the audit was 43\% (25/58). The most commonly prescribed class of antimicrobial agents was penicillin/Beta-lactamase inhibitor combinations 39.4\% (13/33).

Conclusions: Findings demonstrate frequent antimicrobial consumption of broad spectrum antimicrobial agents which highlights the importance of expanding antimicrobial stewardship programs in this population.
\end{abstract}

Key words: Antimicrobial therapy; antibiotic prescription; hospital

J Infect Dev Ctries 2013; 7(12):990-993. doi:10.3855/jidc.3126

(Received 05 November 2012 - Accepted 24 February 2013)

Copyright (C) 2013 Hammuda et al. This is an open-access article distributed under the Creative Commons Attribution License, which permits unrestricted use, distribution, and reproduction in any medium, provided the original work is properly cited.

\section{Introduction}

A rise in rates of antimicrobial resistance and a decrease in development of new antimicrobial agents has internationally led to an interest in the development of antimicrobial stewardship programs. The goal of an antimicrobial stewardship program is to optimize patient outcomes while minimizing consequences of antibiotic use [1]. The Infectious Disease Society of America (IDSA) has generated evidence based guidelines suggesting implementation strategies for antimicrobial stewardship programs. The exact combination of interventions which will result in improved patient outcomes is not known [1]. Antimicrobial stewardship programs should be designed to improve patient outcomes through multidisciplinary interventions using a variety of strategies designed to meet the specific needs of the health care institution [2].

One strategy for determination of specific needs within the institutions is completion of a point prevalence survey (PPS). Point prevalence surveys have been used internationally to characterize the use of antibiotics within and across institutions [3-9]. This type of audit is useful to identify changes in prescribing practices over time and differences in prescribing practices between institutions [3]. Point prevalence surveys are also useful for identification of targets for quality improvement and evaluation of the effectiveness of antimicrobial stewardship programs within an institution [4].

Literature describing antimicrobial stewardship programs in Middle Eastern countries is limited. In order to meet the unique needs of the Qatari population through implementation of an antimicrobial stewardship program, an assessment of current antibiotic utilization is needed. The aim of this study was to determine prevalence of antimicrobial utilization for oncology in Qatar and to characterize the use of antimicrobial agents in this population.

\section{The Study}

Study Design

This study was designed as a chart audit of antimicrobial use in patients admitted to the oncology and hematology wards in Qatar. This study was conducted in accordance with the Declaration of Helsinki and national and institutional standards. Ethics approval was obtained from the Qatar University Institutional Review Board (QU-IRB 114E/12) and Hamad Medical Corporation, Medical Research Center (12059/12). 
Table 1: Baseline Characteristics

\begin{tabular}{lc}
\hline Characteristics & Total N(\%) \\
\hline Gender (Male) & $13(52)$ \\
Age (Mean 57.1 \pm 14.9) & \\
Nationality & $7(28)$ \\
Qatari & $5(20)$ \\
Egyptian & $4(16)$ \\
Pakistani & $9(36)$ \\
Others & $4(16)$ \\
Initial Cancer Diagnosis & $5(20)$ \\
Lymphoma & $16(64)$ \\
Leukemia & \\
Solid Organ Cancer & \\
\hline
\end{tabular}

Table 2: Antimicrobial Utilization

\begin{tabular}{lc}
\hline Prescribed Antimicrobial Class or Agent & N (\%) \\
\hline Penicillins/Beta-Lactamase Inhibitors & $13(39.4)$ \\
Carbapenems & $5(15.2)$ \\
Fluoroquinolones & $3(9.1)$ \\
Cephalosporins & $3(9.1)$ \\
Imidazole derivative & $2(6.1)$ \\
Sulfonamides/Trimethoprim & $2(6.1)$ \\
Penicillins & $2(6.1)$ \\
Aminoglycosides & $1(3.0)$ \\
Oral vancomycin & $1(3.0)$ \\
Macrolides & $1(3.0)$ \\
\hline
\end{tabular}

\section{Data collection}

Data was collected from the National Center for Cancer Care and Research (NCCCR) on 3 separate days over a 2 week period between April 26 and May 3, 2012. Each medical ward was audited over one day using an adapted version of the 2006 ESAC PPS audit tool [9]. Data was collected by two pharmacy students in their final year of training. Approximately $20 \%$ of charts were assessed by a second investigator with advanced training in pharmacotherapy (EB) to assess accuracy of data collection. The following information was collected: age, gender, nationality, allergy status, antimicrobial agent including dose, route, frequency, and duration (classified according to the anatomical therapeutic chemical (ATC) classification system), [10] reason for prescribing the antimicrobial agent (treatment and prophylaxis), and available culture results. Furthermore, an assessment of compliance with local prescribing policies, febrile neutropenia guidelines, and prescribing based on culture results was completed. Data was collected from patients' electronic and paper-based charts.

\section{Study Participants}

On the day of the audit, charts of all patients admitted to NCCCR were reviewed. Adult inpatients receiving systemic antibiotic prescriptions at 8:00 am were included in the audit. Patients receiving antibiotics through outpatient intravenous programs were excluded.

\section{Statistical analysis}

Data was entered into IBM SPSS, Version 21.0, (Armonk, New York, USA). Results were summarized descriptively using means, standard deviations, and frequencies.

A total of 58 inpatient charts were reviewed during the study period. The overall prevalence of antimicrobial use during the audit was 43\% (25/58). Baseline characteristics of patients who were being treated with antimicrobial agents $(\mathrm{n}=25)$ are described in Table 1. All patients included in the review had a diagnosis of cancer.

A total of 33 antimicrobial agents were prescribed to the 25 study participants. Antimicrobial agents were prescribed for treatment of an active infection in $93.9 \%(31 / 33)$ of antimicrobial orders while $6.1 \%$ $(2 / 33)$ were ordered for prophylaxis. The most frequently used class of antimicrobial agents was penicillin/Beta-lactamase inhibitor combinations $39.4 \%$ (13/33), followed by carbapenems $15.2 \%$ $(5 / 33)$. Frequency of prescribed antimicrobial agents 
are outlined in Table 2. The majority of antimicrobials prescribed were given via the intravenous route, $72.7 \%(24 / 33)$.

A reason for prescribing antimicrobials was documented in $81.8 \%(27 / 33)$ of prescribed agents. The most common documented reasons for antimicrobial use were febrile neutropenia $21.2 \%$ $(7 / 33)$, bacteremia $18.2 \%(6 / 33)$ and fever $15.2 \%$ $(5 / 33)$. Planned duration of antimicrobial therapy was reported for $24.2 \%(8 / 33)$ of all antimicrobial orders.

The majority of antimicrobial prescriptions complied with local prescribing restriction guidelines with $57.6 \%(19 / 33)$ of prescriptions ordered by an approved prescriber. Pre-therapy cultures were performed for $96.0 \%(24 / 25)$ of patients. In those patients who had a sensitivity done, $100 \%(6 / 6)$ of antibiotic choices matched sensitivity results. Local febrile neutropenia guideline were implemented according to local prescribing protocols in 33\% (2/6) of patients.

\section{Conclusion}

To the best of our knowledge, this study is the first PPS in the region. PPS studies have been completed internationally in different institutions and patient populations. The prevalence of systemic antimicrobial agents used internationally has been reported to be approximately $29-38 \%$ [3-5,7,9]. Findings from this study indicate $43 \%$ of patients audited were on systemic antibiotics. While the study population in Qatar had a higher prevalence of antimicrobial use, these findings are not unexpected given the immunocompromised population included in this audit.

Results from this study demonstrate several targets for improvement in antimicrobial use. Few patients on antimicrobial therapy had clear documentation the planned duration of therapy. In addition, the majority of patients $(72.7 \%)$ were prescribed intravenous antimicrobial agents. While appropriateness of the route of administration was not accessed, previously completed audits demonstrated lower rates of intravenous antimicrobial prescribing [3-5]. Furthermore, approximately a third of prescribed antimicrobial agents did not follow local antimicrobial prescribing policies at NCCCR. The above targets should be further evaluated and addressed through interdisciplinary collaboration and development of antimicrobial stewardship programs amongst physicians, pharmacists, nurses, microbiologists, and other health care providers.
Several limitations of this study should be considered. All patients included in this audit were immunocompromised and had a primary diagnosis of cancer. As a result, these findings may not reflect antibiotic prescribing practices in other institutions in Qatar. Furthermore, sample size was limited given the specialized population of patients included in this study. Despite these limitations, this study demonstrates use of broad spectrum antimicrobial agents in the inpatient oncology population in Qatar is common. These findings emphasize the importance of development, implementation, and expansion of an antibiotic stewardship program at NCCCR.

\section{Acknowledgements}

We would like to thank Qatar University for financial support in completing this project and the nursing and pharmacy staff at NCCCR for their support and facilitation of data collection.

Findings from this project were presented at the Qatar Foundation Annual Research Forum from October 21st to 23rd, 2012 in Doha, Qatar.

\section{Funding}

This work was supported by an internal student grant from Qatar University (QUST-CPH-SPR-12-4)

\section{References}

1. Dellit TH, Owens RC, McGowan JE, Gerding DN, Weinstein RA, Burke JP, Huskins WC, Paterson DL, Fishman NO, Carpenter CF, Brennan PJ, Billeter M, Hooton TM (2007) Infectious Disease Society of America and the Society for Healthcare Epidemiology of America Guidelines for Developing an Institutional Program to Enhance Antimicrobial Stewardship. Clin Infect Dis 44: 159-177.

2. Patterson D (2006) The Role of Antimicrobial Management Programs in Optimizing Antibiotic Prescribing within Hospitals. Clin Infect Dis 42: S90-5.

3. Seaton RA, Nathwani D, Burton P, McLaughlin C, MacKenzie AR, Dundas S, Ziglam H, Gourlay Y, Beard K, Douglas A (2007) Point prevalence survey of antimicrobial use in Scottish hospitals utilizing the Glasgow Antimicrobial Audit Tool (GAAT). Int J Antimicrob Agents 29: 693-699.

4. Naughton C, Hennessy Y, Mannion C, Philbin M (2011) A comparison of antibiotic point prevalence survey data from four Irish regional/general hospitals. Ir J Med Sci 180: 457461.

5. Zarb P, Amadeo B, Muller A, Drapier N, Vankerckhoven V, Davey P, Goossens H (2011) Identification of targets for quality improvement in antimicrobial prescribing: the webbased ESAC Point Prevalence Survey 2009. J Antimicrob Chemother 66: 443-449.

6. Aldeyab MA, Kearney MP, McElnay JC, Magee FA, Conlon G, Gill D, Davey P, Muller A, Goossens H, Scott MG (2011) A point prevalence survey of antibiotic prescriptions: benchmarking and patterns of use. Br J Clin Pharmacol 71: 293-6. 
7. Amadeo B, Zarb P, Muller A, Drapier N, Vankerckhoven V, Rogues AM, Davey P, Goossens H (2010) European Surveillance of Antibiotic Consumption (ESAC) point prevalence survey 2008: paediatric antimicrobial prescribing in 32 hospitals of 21 European countries. J Antimicrob Chemother 65: 2247-2252.

8. Zarb P, Goossens H (2011) European Surveillance of Antimicrobial Consumption (ESAC): Value of a PointPrevalence Survey of Antimicrobial Use Across Europe. Drugs 71: 745-755.

9. Ansari F, Erntell M, Goossens H, Davey P (2009) The European Surveillance of Antimicrobial Consumptions (ESAC) Point-Prevalence Survey of Antimicrobial Use in 20 European Hospitals in 2006. Clin Infect Dis 9: 1496-1504.
10. WHO Collaborating Center for Drug Statistics Methodology, Guidelines for ATC Classification and DDD Assignment 2012. Oslo, 2011.

\section{Corresponding author}

Dr. Emily Black

Qatar University, College of Pharmacy

PO Box 2713

Doha, Qatar

Phone: +974 4403 5578, Fax: +974 44035551

Email: Emily@qu.edu.qa

Conflict of interests: No conflict of interests is declared. 